\title{
QoS Provisioning for Large-scale Multi-AP WLANs
}

\author{
Jaehyuk Choi ${ }^{\mathrm{a}, *}$, Kang G. Shin ${ }^{\mathrm{a}}$ \\ ${ }^{a}$ Department of Electrical Engineering and Computer Science, \\ University of Michigan, Ann Arbor, MI 48109-2122, U.S.A.
}

\begin{abstract}
Large-scale deployment of IEEE 802.11 wireless LANs (WLANs) with a high density of access points (APs) has become commonplace due mainly to its potential for numerous benefits, such as ubiquitous service coverage, seamless handover, and improved link quality. However, the increased AP density can induce significant channel contention among neighboring cells, thus causing severe performance degradation and throughput imbalance between cells. There have been a plethora of research efforts to improve the WLAN performance, but most of them focused only on single WLAN environments without accounting for intercell contention. The de facto QoS provisioning mechanism for WLANs, i.e., the Enhanced Distributed Channel Access (EDCA), is no exception to this. The EDCA focuses only on inter-flow priority distinction and has not considered the effect of inter-cell contention which significantly restricts its efficiency. This paper presents an enhanced QoS provisioning framework that takes into account inter-cell level differentiation as well as inter-flow level priority, which may be viewed as extension of QoS provisioning from a single-WLAN domain to a multiWLAN domain. We also propose an architecture for managing multi-AP systems in which a central controller regulates the wireless channel occupancy of APs by adaptively configuring the cell-level QoS parameters. Our extensive simulation results show that the proposed inter-AP cooperative QoS scheme overcomes the limit of legacy 802.11e and provides a high level of fairness in large-scale denselydeployed WLANs.
\end{abstract}

*Corresponding author. Department of Electrical Engineering and Computer Science, University of Michigan, 2260 Hayward St., Ann Arbor, MI 48109-2121, USA. Fax: +1 (734) 763-8094. E-mail address: jaehchoi@umich.edu (Jaehyuk Choi).

Email addresses: jaehchoi@umich . edu (Jaehyuk Choi), kgshin@eecs. umich. edu (Kang G. Shin) 
Keywords: IEEE 802.11e; QoS Provisioning; Large-scale WLAN; Centralized QoS Architecture.

\section{Introduction}

Large-scale deployment of 802.11 WLANs with a high density of access points (APs) has received significant attention in recent years with rapidly increasing use of mobile computing devices, such as laptops, PDAs, and Wi-Fi-enabled phones $[19,34]$. Such WLANs have become wide-spread, common enterprise and university campus networks [35]. The high-density deployment of APs has potential for numerous benefits including ubiquitous service coverage, seamless handover, and improved link quality to the clients $[22,35]$. Despite their potential merits, however, the high-density WLANs suffer from severe performance degradation and unfair throughput imbalance due to significant channel contention among cochannel neighboring cells [7, 19,34]. The performance imbalance problem is mainly due to the fundamental limitation of CSMA-based random access mechanism, which gives preference in media access to some links over others depending on their spatial locations $[23,16]$. Although the inter-cell channel contention and interference can be reduced by allocating orthogonal channels to neighbor cells $[19,28,22,17,34]$, only 3 orthogonal channels are available in the $2.4 \mathrm{GHz}$ band, which is not enough to eliminate the co-channel interference. Consequently, the inter-cell channel contention and interface are inevitable in such the multi-cell environment with high AP densities.

However, most prior research efforts to improve system performance for 802.11 WLANs focused only on single WLAN environments [12, 30, 21, 18, 31, 38]. They did not account for the impact of high AP-density on system, overlooking the inevitable inter-cell channel contention and thus restricting their efficiency and providing poor performance in many multi-cell scenarios.

The quality-of-service (QoS) provisioning mechanism for WLANs is no exception. The de facto QoS standard, i.e., the 802.11 Enhanced Distributed Channel Access (EDCA) [2], focuses only on inter-flow priority distinction within a single-cell WLAN without considering the significant impact of inter-cell channel contention. To ensure QoS guarantees for real-time applications such as voiceover-IP, it is essential that the EDCA provides stable channel access opportunities to nodes with low channel access delay. However, the EDCA is inherently based on the CSMA random access protocol and thus can easily suffer from the severe unfairness among neighboring cells. For example, our simulation result shows 
that the QoS guarantee for flows in a cell - whose spatial location is disadvantageous than neighboring cells in terms of channel sensing - is hardly achieved by the EDCA due to lack of transmission opportunities even when its intra-cell channel contention is moderate $[37,13,16,20]$.

In this paper, we present a comprehensive QoS support mechanism that overcomes the limitation of current QoS scheme and resolves the throughput imbalance among Basic Service Sets (BSSs) in dense large-scale 802.11 WLANs. To meet this goal, we discuss how the current 802.11 QoS provisioning mechanism should be modified for multi-WLAN systems, and introduce an enhanced QoS provisioning framework that takes into account the inter-BSS level differentiation as well as inter-flow prioritization within a intra BSS by extending the 802.11 EDCA. Our approach may be viewed as an extension of QoS provisioning from a single-WLAN domain to a multi-WLAN domain. In addition, We present a QoS architecture for managing multi-WLAN systems, in which a central controller arbitrates the wireless channel occupancy of APs. The heart of our QoS framework is that it has the ability of inter-BSS differentiation and thus, allows the system to regulate the inter-cell channel contention so as to support QoS provisioning.

The centralized solutions/architectures for WLANs have already been popular and used widely in many large-scale networks such as enterprise and campus networks [35], in which the entire APs are centrally managed and their parameters, such as the channels and the transmit powers, are configured by a central control element. Thus, the proposed QoS framework is easy to be deployed in today's large-scale WLAN architectures. It does not require any additional software or any changes at mobile clients.

We have evaluated the effectiveness of the proposed scheme via extensive simulations. The simulation results show that the proposed QoS scheme overcomes the limit of legacy 802.11e in dense multi-AP systems.

The remainder of the paper is organized as follows. Section 2 discusses the related work, positioning our work in a comparative perspective. Section 3 describes the background of this work and the problem addressed in this paper. We present an enhanced WLAN QoS provisioning framework and a QoS architecture in Section 4. In Section 5, we propose an dynamic inter-cell differentiation algorithm. We evaluate the performance of the proposed framework using extensive simulations in Section 6. The paper concludes with Section 7. 


\section{Related Work}

Many studies have already identified the channel unfair share and the flow starvation problem in 802.11-based wireless ad hoc networks $[37,13,16,20]$. There have also been numerous efforts to mitigate the inter-cell interference problem, including channel assignment [17, 28, 34], power control (or carrier sense control) $[8,26,36]$, and association control $[9,10]$.

Channel Assignment: Many commercial AP vendors implement a simple distributed scheme commonly called the least congested channel search (LCCS) [17]. In LCCS, upon its initialization, the AP scans the channels in its vicinity and selects the least congested channel. Mishra et al. in [27] formulated the channel assignment as a weighted vertex coloring problem. The authors of [28] addressed the problem of distributed channel assignment in uncoordinated wireless environments, and proposed a dynamic channel hopping protocol that distributively assig the channel of an AP. It provides good fairness and can also take full advantage of partially-overlapped channels for a throughput gain in densely-deployed networks. A recent study in [34] considered the traffic pattern for the selection of channels, showing that traffic-aware channel assignment can significantly improve the quality of a channel assignment.

Power Control: The second approach is power control or adaptive control of carrier sensing ranges. Proper management of transmission power reduces interference and thus improves the overall network performance. In [8], Akella et al. presented a power-control and rate-selection (called PERF) algorithm. The PERF algorithm reduces the transmission power as much as possible without reducing the transmission rate, thus improving aggregate throughput. Recently, the work described in [26] showed that the problem of power control in 802.11 networks requires the joint optimization of transmit power and CCA thresholds across the network. The ECHOS algorithm in [36] aims to improve the capacity of the IEEE 802.11 hotspots by dynamically adjusting the CS threshold (CST). Existing settings for CST in 802.11 networks are shown to be very conservative. Therefore, transmitters dynamically choose their CST values according to on their signal strength at the receiver. This dynamic selection of CST is shown to make a significant improvement in the performance of hotspot networks.

Association Control: Association control is used to balance client-association across a set of APs $[9,10]$. The results in [30,9] have shown that the loadimbalance problem can be alleviated by balancing the load among the APs by intelligently selecting the user-AP association. The authors of [9] proposed a load-balancing algorithm to associate a new user with the AP that can provide a 
minimal bandwidth required by the user. If there are more than one such AP, the one with the strongest signal is selected. The authors of [22] proposed an algorithm by jointly considering channel selection and client association. It uses the Gibbs sampler framework and presents fully-distributed algorithms for (i) channel selection to mitigate interference and (ii) user association for fair and optimal sharing of bandwidth among users.

Although these approaches can reduce inter-cell interference and can thus be effective to improve the overall network performance, they are not suitable to deal with the MAC-layer congestion. That is, they are mostly based on long-term decisions and thus not agile enough to infer and react to the immediate and bursty nature of network congestion. For these reasons, we want to develop a mechanism that can address the network congestion quickly and mitigate the throughput imbalance among BSSs, which are orthogonal and hence complementary to existing techniques, such as channel assignment, power control, and association control.

\section{Background and Motivation}

In this section, we first describe the legacy 802.11e EDCA protocol briefly. Then, we point out the limitations of EDCA in densely-deployed large-scale WLANs via a density analysis using a real AP deployments data set.

\subsection{IEEE 802.11e: The Current QoS Support Mechanism}

The IEEE 802.11e standard [2] defines a set of Quality of Service enhancements for WLAN through a new coordination function, namely Enhanced Distributed Channel Access (EDCA). EDCA provides service differentiation for QoS data by controlling medium access and classifying traffic with four Access Categories (ACs). Each AC contends for the medium access separately via an Enhanced Distributed Channel Access Function (EDCAF) with a different priority. Priority differentiation among ACs is achieved by differentiating the channel access parameters, namely, QoS Parameter Set Element (QPSE). It defines, for AC $i(i=0, \cdots, 3)$, the arbitration inter-frame space AIFS $[i]$, the initial contention window size $C W_{\min }[i]$, the maximum contention window size $C W_{\max }[i]$, and the Transmission Opportunity TXOP $[i]$. These parameters are announced by the QoS Access Point (QAP) periodically via beacon frames, and can be adaptively adjusted by the QAP based on the network traffic condition. Different values of these parameters enforce the traffic of different priorities to spend different amounts of time for initiating their transmissions, so as to differentiate their probability of 
medium access. For example, if one AC has a smaller AIFS (or $C W_{\text {min }}$ ), the AC's traffic has a better chance to access the wireless medium earlier.

Despite the several enhancement features of EDCA over the legacy 802.11 DCF, the EDCA still suffers from similar performance problems in the legacy DCF-based WLANs because its operation is essentially same as that of DCF [20]. In particular, it has been shown that both EDCA and DCF do not utilize their maximum potential capacity, and their throughput performance and degree of fairness degrade significantly as the number of contending stations increases [21]. A more general problem with EDCA is that it inherently involves the unfairness problem stemming from the CSMA-based medium access rule $[23,16]$.

\subsection{Neighboring Channel Contention in Large-scale WLANs}

To understand the deployment state of real large-scale networks, we now investigate the AP deployment state of a centrally-managed campus network [24]. We have analyzed campus network data sets obtained from Dartmouth's $C R A W$ $D A D$ project database [24]. The data set includes a list of APs, its coordinates and the average signal strength from each of the other APs.

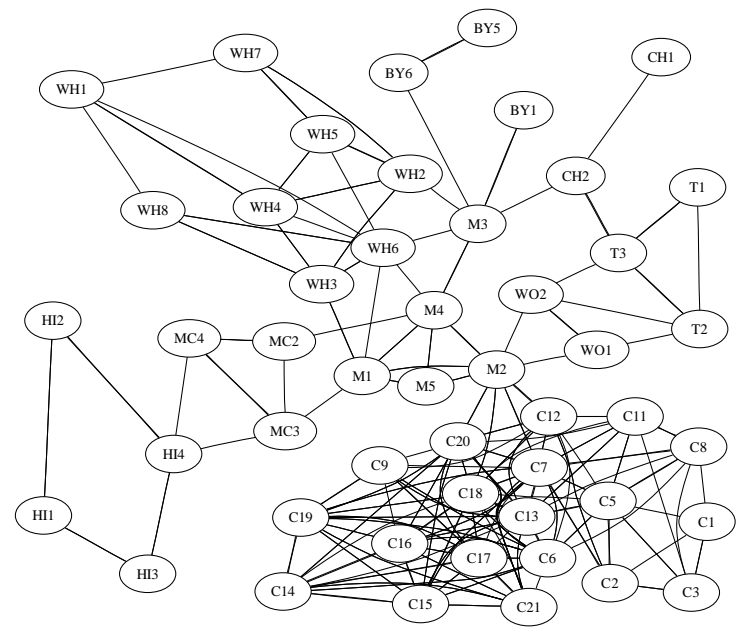

Figure 1: AP connectivity graph; the largest connected clique (size of 52) observed in the Dartmouth's campus network data set in 2004.

Fig. 1 shows the AP connectivity graph for a connected clique observed on the Dartmouth campus. The edges in the graph represent the connectivity information between two APs where the APs can hear the transmission of each other if they transmit on the same channel. From the result, we can observe exceedingly high 
AP connectivity, which implies that the APs' contention for use of the medium is intense, even though the campus network has been 'centrally' managed. Although the co-channel inter-cell channel contention can be reduced by allocating nonoverlapping channels to neighbor cells [19, 28, 22, 17, 34], only 3 non-overlapping channels are available in the $2.4 \mathrm{GHz}$ band, which is not enough to avoid the interference in such a high AP density environment. Consequently, it can be easily shown that, even if the optimal channel arrangement is adopted, these APs will be in the transmission range of at least one other AP in their vicinity in Fig. 1. Thus, each AP can sense transmissions of neighbor APs and should contend with the neighbor APs and their client nodes for the channel access.

In summary, the co-channel inter-cell channel contention in such the multi-cell environment with high density of APs is inevitable and commonplace, and thus its impact on the performance of the current 802.11 QoS-provisioning mechanism, which currently does not account for inter-cell channel contention, needs to be understood to design a more flexible and efficient QoS support mechanism.

\subsection{Limitation of 802.11e QoS Mechanism}

We now use simple $n s-2$ simulations to show the impact of co-channel channel contention among neighboring cells on the performance of channel differentiation of the original EDCA mechanism. We have tested on two simple scenarios with single-WLAN and three contending WLAN topologies shown in Fig. 2. To see the impact of inter-cell channel contention, the simulation scenarios focus on a co-channel environment where all the APs and member nodes are in a same channel. Actually, in the real campus network shown in Fig. 1, we can observe several connected cliques whose AP connectivities correspond to the topology with three contending APs shown in Fig. 2(b) when the optimal channel assignment is applied. We consider three flows in the two scenarios where each flow is set to perform TCP downloading for various conditions of offered load (Mbps); the uplink traffic is TCP-ACKs.

Figs. 3 show the throughput performance as a function of offered load for single cell and three cell scenarios, with emphasis on the EDCA's priority differentiation mechanism. Fig. 3(a) shows that EDCA provides per-flow priority effectively in a single-cell environment. In other words, a higher-priority flow (i.e., class 1) achieves higher throughput than the other (lower-priority) flows even when the network is saturated.

However, in the multi-cell environment shown in Fig. 3(b), we can observe the limitation of EDCA protocol, that is, the throughput of the middle flow $\mathrm{AP}_{0^{-}}$

$\mathrm{c}_{0}$ decreases to almost zero when the offered load of outer flows is larger than a 


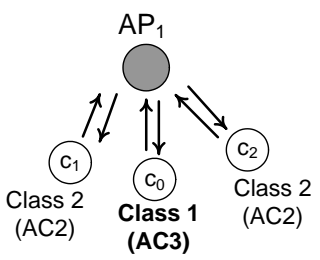

(a) a single cell

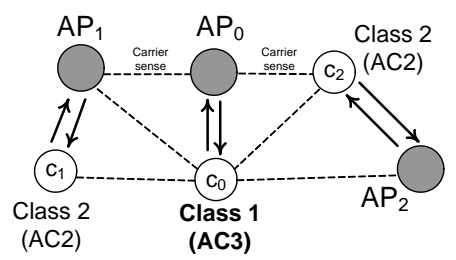

(b) three co-channel contending cells

Figure 2: Simple simulation topologies with (a) a single-cell WLAN (b) a multi-cell WLAN

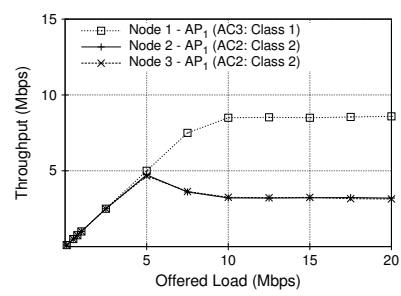

(a) Single-cell environment

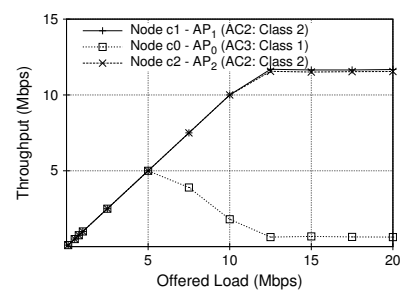

(b) Multi-cell (FIM) environment

Figure 3: Throughput per class vs. offered load for 802.11e EDCA in (a) a single WLAN environment and (b) a multi-cell environment with 802.11g PHY parameters (no channel error). Minimum and maximum Contention Windows $(\mathrm{CW})$ are configured as $C W_{1} \in[15,63]$ for class 1 , and $C W_{2} \in[31,1027]$ for class 2 in the simulation.

certain value even though its priority (class 1) is higher than all the other outer flows. The source of the high-priority middle flow' starvation is due to lack of its transmission opportunities. Particularly, due to its spatial location, the middle flow $\left(\mathrm{AP}_{0}-\mathrm{c}_{0}\right)$ can access the medium only when both outer neighbor flows are idle. However, the probability of the two outer flows being idle at the same time is very low because the outer flows' transmissions are asynchronous and overlap randomly at the middle flow. As a result, the middle flow will almost always sense the medium as being busy and hence, defer its transmission continuously, thus suffering from starvation. This problem has been already identified in 802.11 DCF-based wireless ad hoc networks (see for example [37, 13, 16, 20]), referred as Flow-in-the-Middle (FIM). As shown in the simulation result in Fig. 3, this unfairness problem also severely restricts the efficiency of EDCA's prioritized channel access mechanism.

In summary, the priority scheme of EDCA, based on the differentiated access probabilities, has the CSMA's inherent weakness of unfair channel share. As a 
result, the EDCA's mechanism for QoS support can suffer from the severe unfairness among neighboring cells in densely-deployed WLANs in which the inter-cell channel contention is unavoidable.

\section{A QoS-Provisioning Framework with Cooperative 802.11e EDCA for Multi- AP Systems}

Our objective is to develop a comprehensive QoS support mechanism that can overcome the limitation of current QoS-provisioning and address the throughput imbalance between BSSs in multi-WLAN systems. We first discuss how the current 802.11 QoS-provisioning mechanism should be modified for multi-cell environments, which currently does not account for inter-cell interference. Specifically, we propose a new QoS-provisioning framework that enhances the current 802.11e standard, by considering the inter-BSS level differentiation as well as inter-flow level prioritization in an intra-BSS. We also present an architecture in which a central controller configures the intra- and inter-cell QoS parameters dynamically.

\subsection{Extension of EDCA for Intra and Inter-Cell QoS Provisioning}

The basic EDCA is designed to support the MAC-level prioritized QoS for time-bounded multimedia applications such as voice and video in the IEEE 802.11 WLAN. The prioritized access among traffic classes (ACs) is achieved by differentiating the channel access parameters, namely, QPSE. However, the differentiation in priority among ACs only focuses on inter-flow priority distinction within a single-cell WLAN. It does not address the inter-cell channel contention even though the inter-cell contention greatly affects the MAC-layer performance critical to QoS provisioning.

Our objective here is to develop an appropriate way to provide the required QoS guarantee in densely-deployed WLANs in which the inter-cell contention is unavoidable. Obviously, it basically requires robustness against inter-cell channel contention and thus it must be able to handle the inter-cell throughput imbalance among neighboring contending BSSs. Our main idea is that the priority differentiation is required not only for inter-flow level within a BSS but also for inter-BSS level. To realize this idea for inter-BSS differentiation in prioritizing channel access, we introduce the following additional inter-BSS coordination parameters assigned to each BSS. We will refer to these parameters as Inter-BSS QoS Parameter Set Element or BQPSE. 
- BSS Interframe Space (BIFS): The additional minimum time interval for the medium to remain idle before starting a backoff.

- BSS Minimum Contention Window $\left(B C W_{\min }\right)$ : The additional minimum Contention Window basis.

- BSS Transmission Opportunity (BTXOP) limit: The maximum duration for which a node can transmit after acquiring access to the channel considered in a given BSS.

In the proposed method, each QAP updates its basic EDCA parameters by involving BQPSE for all four ACs $(i=0, \cdots, 3)$ as follows:

- $A I F S[i] \leftarrow A I F S[i]+$ BIFS

- $C W_{\min }[i] \leftarrow C W_{\min }[i]+\mathbf{B C} \mathbf{W}_{\min }$

- $T X O P[i] \leftarrow T X O P[i]+$ BTXOP.

Unlike the basic EDCA parameters having different values for four ACs, BQPSE is an AC independent BSS-level parameter and the same value is assigned to all the ACs. The rational behind this is to arbitrate the imbalance of transmission opportunities among BSSs while keeping the inter-flow priority configuration in each individual QAP. Note that although our approach requires additional consideration for BSS-dependent parameters (BQPSE), they are incorporated into basic EDCA parameters (i.e., QPSE) when announced via beacon frames. Thus, clients or QoS stations (QSTAs) associated with the QAP do not require to be aware of BQPSE, that is, no client-side modification is required.

One can use several different ways to determine the BQPSE parameters; (i) control by a central authority, (ii) distributed coordination among QAPs, and (iii) self-adjustment by individual QAPs. In the following sections, we consider the first approach, i.e., centrally-managed networks like campus and enterprise networks. We present an architecture that determines the BQPSE parameter by interQAP coordination achieved by an inter-QAP communication protocol.

\subsection{QoS Architecture}

In general, 802.11 WLAN deployments can be classified into two categories [8], depending on whether to be managed centrally or not: (i) centralized deployments and (ii) uncoordinated deployments (also referred to as 'chaotic' deployments [8]). In the former architecture, the entire network is observed and managed 
by a central control element. Such architecture provides several benefits such as ease of management and better design of various control and security functions. For these reasons, the centralized architecture has been adopted and deployed widely in lots of enterprise and university campus networks. This work primarily

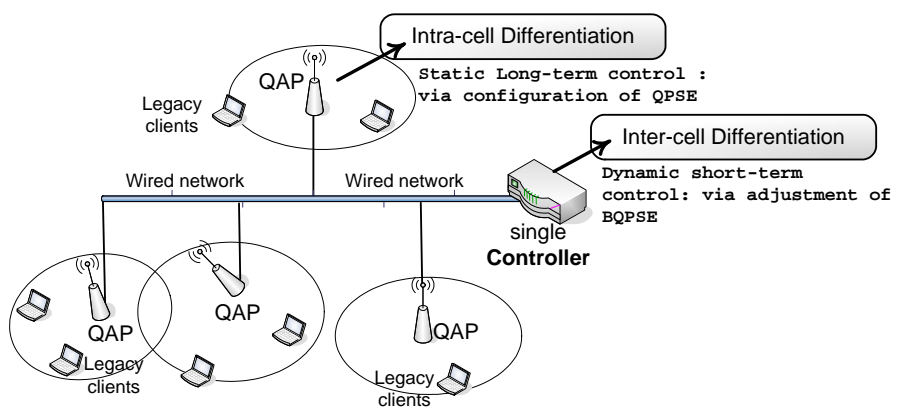

Figure 4: Illustration of centralized QoS-provisioning architecture

considers the centrally-administrated networks in which a central controller configures the BSS-level QoS parameter (i.e., BQPSE) dynamically to regulate the inter-cell channel contention.

Fig. 4 provides a high-level illustration of the proposed QoS-control framework where a centralized controller manages the 802.11e QoS parameters of entire QAPs in a WLAN system. It configures two types of QoS parameters: QPSE for intra-cell per-flow prioritization and BQPSE for inter-cell prioritization. Configuration of intra- and inter-cell QoS parameters is orthogonal to each other. In other words, policies for inter-cell QoS parameters can be combined with various other previously-proposed QPSE adjustment schemes including the default static approach in the standard [2], traffic-aware configuration [37], and so on. In general, the intra-cell QoS parameters QPSE are fixed or updated infrequently, i.e., managed based on a long-term update policy. In contrast, the BSS-level QoS parameter BQPSE is adjusted repeatedly by the controller in order to regulate the immediate and dynamic inter-cell channel contention, i.e., managed based on a short-term control policy.

The proposed QoS architecture provides flexibility for the system to regulate the inter- and intra-cell channel contention so as to provide QoS provisioning. Thus, many different possible policies can be adopted to determine or adjust the QPSE and BQPSE parameters for various purposes with respect to intra- and intercell priority distinction, respectively. 


\section{Adaptive Inter-QAP Contention Control for QoS Provisioning}

The heart of the proposed QoS framework is its ability of inter-BSS differentiation allowing us to regulate the inter-cell channel contention. In this section, using the QoS framework, we propose an adaptive contention control algorithm to improve inter-AP fairness which is essential to support QoS in large-scale WLAN systems.

\subsection{Measurement of Neighboring Interference Between APs}

The first step for inter-cell contention control is to acquire channel contention information among QAPs (QoS APs). Given a set of deployed APs, the multiAP system can be represented as a inter-AP contention graph. This graph can represent the neighboring interference among APs, i.e., which AP is contending with which other APs.

We define the inter-AP contention graph as a directed graph $C=(V, G)$ where $V=\left\{A P_{1}, A P_{2}, \ldots, A P_{n}\right\}$ is the set of all considered APs, $G$ is a set of edges representing the neighboring contentions between APs. There is a directional edge $g_{i j} \in G$ from $A P_{i}$ to $A P_{j}$ if there is neighboring interference from $A P_{i}$ to $A P_{j}$, where $g_{i j}$ ranges in $[0,1]$. The cost of edge, $g_{i j}$, can be used to characterize the channel contention relation between two APs, and can be classified into three types: (i) complete neighboring AP, (ii) partial neighboring AP, and (iii) independent AP. If $A P_{i}$ is within the physical or virtual carrier sensing range of $A P_{j}$, $A P_{i}$ is a complete neighboring AP of $A P_{j}$ and has the value of $g_{i j}=1$. If $A P_{i}$ is outside of $A P_{j}$ 's carrier sensing range but within the sensing range of the transmissions of clients associated with $A P_{j}, A P_{i}$ is a partial neighboring AP of $A P_{j}$ and the edge cost is $0<g_{i j}<1$. For the other cases, we define it as an independent AP to each other and $g_{i j}=0$. Let us define $H_{i}=\left\{A P_{k}: A P_{k} \in V,\left(g_{i j}>0\right) \in G\right\}$, i.e., it denotes the set of all neighbor APs of $A P_{i}$ including both complete and partial neighboring APs in $G$. The notation of $\left|H_{i}\right|$ represents the number of $A P_{i}$ 's interfering APs. The neighboring interference edge $g$ between two APs depends on the placement of APs, currently-used channel number, signal strength and other topological factors, and in most case, the geographical distance between APs. For example, even though $A P_{i}$ and $A P_{j}$ are located very close to each other, $g_{i j}=g_{j i}=0$ if their channels of $A P_{i}$ and $A P_{j}$ are orthogonal.

For the generation of inter-AP contention graph $C=(V, G)$, we assume that the inter-AP contention graph is readily known or can be constructed with the support of many existing probing mechanisms, without developing a new solution. This is a reasonable assumption since many advanced solutions are readily 
available for the construction of contention graphs based on active probing, passive probing and hybrid approaches [5, 7, 32, 33, 6]. See [6] for a good survey. The main idea of those approaches is that each sender sends a series of broadcast probes, and all other nodes measure the received signal strength. Then, the other nodes estimate the sending rate based on received signal strength and the carrier sense threshold, and estimate the delivery rate based on SNR. This way, $O(N)$ broadcast probes are required to measure interference in an $N$-node network.

\subsection{Adaptive Control for Managing Inter-cell Contention}

Now, we present a dynamic adaptive control algorithm with which the centralized controller can dynamically configure the cell-level QoS parameter, QBPSE, to arbitrate the channel access among QAPs.

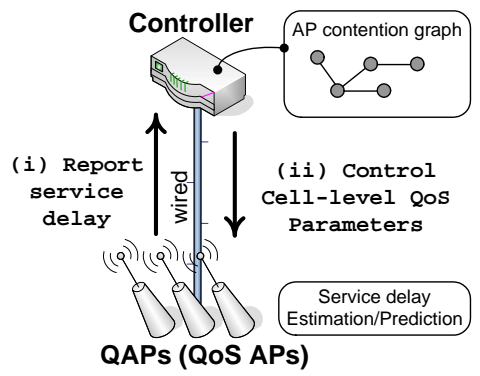

Figure 5: Overview of dynamic control of inter-cell QoS parameters

\subsubsection{Overview}

We describe the ideal form of the algorithm, assuming no propagation delay and allowing message passing delay. The overview of the control algorithm is depicted in Fig. 5.

1. During $t$-th time period whose duration is $\Delta$, each QAP estimates the average queueing delay $Q_{i}(t)$ and the average packet arrival rate $\lambda_{i}(t)$ at its downlink queue of its QAP explicitly. The average queueing delay $Q_{i}(t)$ is calculated by multiplying the measured average service time $T_{s}(t)$ by the current queue length qlen $(t)$, i.e., $Q_{i}(t)=T_{s}(t) \cdot$ qlen $_{i}(t)$. The average packet-arrival rate is measured by dividing the number of total packets arrived during the last time period by $\Delta$ at the end of $t$-th time period. 
2. $Q A P_{i}$ predicts the future queueing delay at the next time period (i.e., $t+1$ th) based on the current measured information as:

$$
\widetilde{Q}_{i}(t+1)=Q_{i}(t)+\kappa\left[\widetilde{\lambda}_{i}(t+1)-1 / T_{s}(t)\right],
$$

where $\kappa$ is $\kappa=\Delta \cdot T_{s}$ and $\widetilde{\lambda}_{i}(t+1)$ is the predicted traffic demand at the next time period.

3. This predicted queueing delay $\widetilde{Q}_{i}(t+1)$ at the next time period is passed to the central controller through the wired network.

4. The controller adjusts the BQPSE for $Q A P_{i}$ based on $\widetilde{Q}_{i}(t+1)$ and the received $\widetilde{Q}_{j}(t+1)$ such that $j \in H_{i}$, and passes the updated BQPSE to $Q A P_{i}$.

5. $Q A P_{i}$ updates EDCA parameters by involving BQPSE and announces it to its clients (QoS stations or QSTAs) via the next transmitted beacon frame.

6. The QAP and its QTAs contend the channel access with the updated EDCA parameter during the next contention period.

For the traffic demand prediction of $\widetilde{\lambda}_{i}(t+1)$ in Step 2, we rely on the traffic history observed during last $W$ time periods, i.e., from $t-W+1$ to $t$-th periods. Formally, we calculate $\tilde{\lambda}_{i}(t+1)$ as

$$
\widetilde{\lambda}_{i}(t+1)=\frac{\max _{s \in[t-W+1, t]} \lambda_{i}(s)+\lambda_{i}(t)}{2},
$$

where $Q A P_{i}$ estimates its future traffic demand as the medium value between the greatest observed traffic during last $W$ periods and the very last offered traffic value $\lambda_{i}(t)$. In the simulation, the duration of the update time period $\Delta$ is set to $100 \mathrm{~ms}$, that is, a general beacon period.

The EDCA parameters, i.e., QPSE, announced by a QAP via a periodic transmitted beacon frame provide to QSTAs the control information for their proper operation of the QoS facility during the next contention period (until the next beacon announcement). In our scheme, the inter-BSS QoS parameters, BQPSE, are absorbed into the QPSE values, and thus, QSTAs do not require to be aware of BQPSE values.

\subsubsection{Adjustment of Inter-BSS QoS parameters}

Now, we specify the control procedure for the control of $Q A P_{i}$. After the controller receives all the periodic reports $\widetilde{Q}_{j}(t+1)$ from QAPs $j \in H_{i}$, it calculates 
the following reference value $\Gamma_{i}$ for $Q A P_{i}$ using the received reports:

$$
\Gamma_{i}=\sum_{A P_{j} \in H_{i}} g_{i j} \widetilde{Q}_{j}(t+1) /\left|H_{i}\right| .
$$

$\Gamma_{i}$ is a weighted sum of neighbor QAP's future queueing delays $\widetilde{Q}_{j}(t+1)$, where the wights are the degree of inter-AP contention from $A P_{i}$ to $A P_{j}$, i.e., edge cost $g_{i j}$. With $\Gamma_{i}$, the control algorithm in $Q A P_{i}$ compares its local delay $\widetilde{Q}_{i}(t+1)$ with $\Gamma_{i}$ and adopts BQPSE parameters according to the comparison result. As the primary means to control the channel access, we use BIFS among several BQPSE parameters [11]. This is because inter-frame space (IFS) differentiation is shown to be more effective than contention window differentiation [11]. However, other parameters including $\mathrm{BCW}_{\min }$ and BTXOP also can be readily used.

When the BSS is determined to occupy the channel much more than others (i.e., $\left.\widetilde{Q}_{j}(t+1)<\Gamma_{i}\right)$, it increases the value of BIFS within the bound of $\mathrm{BIFS}_{\max }$, and vice versa. With the higher value of BIFS duration, the corresponding QAP and its QSTAs require more idle time to access the channel, thereby delaying the channel access time and offering a higher channel access chance to contending stations in nearby BSSs.

In order to satisfy the the requirements of convergence to efficiency and fairness among BSSs, we adopt the additive increase/multiplicative decrease (AIMD) control algorithm. The AIMD algorithm is known to have the property of converging to equal values of the control variable [14]. For example, the window adjustment is made based on the AIMD algorithm, which allows for fair resource allocation and efficient resource utilization [14]. AIMD has been used as the core of all transport protocols that support congestion control [15]. Our control algorithm aiming to achieve fairness among BSSs increases the idle time before attempting the channel by increasing BIFS additively if the BSS is determined to dominate the channel use, while it decreases BIFS multiplicatively when the BSS loses in channel contention with other contending BSSs. Its detail algorithm is given in Algorithm 1.

In the control, the additive increase ratio is determined by the factor $\beta$ and the multiplicative decrease ratio is determined by the factor $\epsilon$. For $\beta$, we use a multiple number of the backoff slot time, aSlotTime $b_{\text {slot }}$ of 802.11 MAC to act as a practical influencing inter-frame spacing value. Thus, we set $\beta=2 \cdot b_{\text {slot }}$ in our performance evaluation. For a quick channel access when a BSS is determined to starve, we use a multiplicative decrease ratio of $1 / 2$ of the current BIFS value (i.e., $\epsilon=0.5$ ). The control parameters are set as follows: 


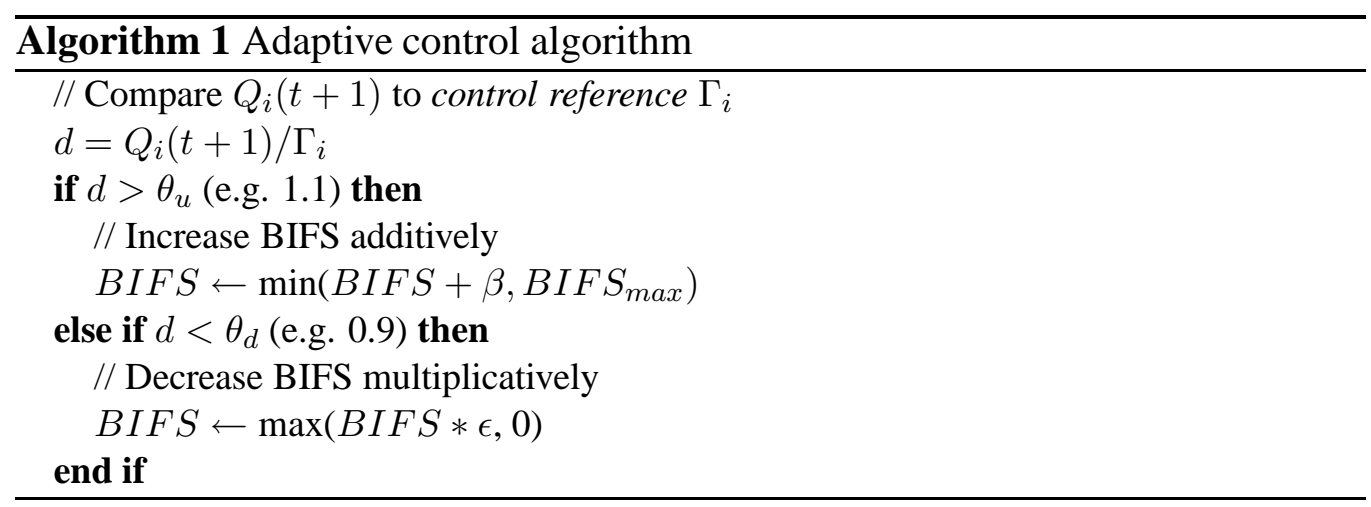

- Increasing/decreasing threshold: $\theta_{u}=\theta_{d}=1.0$

- Increasing factor: $\beta=2 \cdot b_{\text {slot }}(=40 \mu \mathrm{s})$, where $\sigma$ is a slot duration of 802.11

- Decreasing factor: $\epsilon=0.5$

In Algorithm 1, one can see that the value of BIFS is bounded by BIF $S_{\max }$. The factor $B I F S_{\max }$ plays an important role to control the degree of fairness among BSSs since BIF $S_{\max }$ determines the duration of maximum idle time that impacts the channel access probabilities of nearby BSSs significantly. Thus, a larger value of $B I F S_{\max }$ is more likely to achieve a higher degree of fairness (a higher value of BIFS duration implies a higher channel access chance of contending BSSs). However, a little bit more of BIF $S_{\max }$ may decrease the overall network utilization as there is a trade-off between network utilization and fairness [25]. For this reason, we set $B I F S_{\max }=10 \cdot b_{\text {slot }}$. In the next section, we study the effect of $B I F S_{\max }$ on the degree of fairness as well as system throughput.

\section{Performance Evaluation}

In this section, we evaluate the proposed management framework using extensive $n s-2$ simulation [3] on several real hotspot topologies. We have conducted three sets of simulations: (i) basic simulations with six topologies derived from real hotspot topologies derived from Dartmouth database [24] and Wigle data set [4], (ii) a large-scale 802.11 wireless system, and (iii) QoS simulations with Voice-over-IP traffic. Since our approach is orthogonal to channel assignment, these simulations only consider the contention problem in a single channel environment. Note that our framework can operate with various frequency-selection methods that have been proposed before [19, 28, 22, 17]. 
Simulation Setup: For the simulation, we consider an $802.11 \mathrm{~b}$ WLAN environment. The carrier sensing range is set to $550 \mathrm{~m}$, and the transmission range to $250 \mathrm{~m}$. Each AP is set to have the same number of clients (e.g., 4 associated client nodes). We assume that all stations in the system always have pending messages to send where the traffic is generated by FTP applications over TCP flows. The details of simulation parameters are listed in Table 1.

Table 1: Parameters used in simulations

\begin{tabular}{c|c}
\hline Parameter & Value \\
\hline \hline Control time period $\Delta$ & $100 \mathrm{msec}$ \\
Carrier Sensing Range & $550 \mathrm{~m}$ \\
Transmission Range & $250 \mathrm{~m}$ \\
aSlotTime $\left(b_{\text {slot }}\right)$ & $20 \mu \mathrm{sec}$ \\
SIFS & $10 \mu \mathrm{sec}$ \\
DIFS & $50 \mu \mathrm{sec}$ \\
Data rate & $11 \mathrm{Mbps}$ \\
PHY header & $192 \mathrm{bits} / 2 \mathrm{Mbps}$ \\
ACK & $412 \mathrm{bits} / 2 \mathrm{Mbps}+\mathrm{PHY}$ header \\
LongRetryLimit & 4 \\
\hline
\end{tabular}

Hotspot Topologies: We obtained real AP topologies from two popular data sets provided by the Dartmouth CRAWDAD[24] and Wigle[4] database. These data sets provide lists of APs and the topology information, i.e., location, of APs. We have sampled 6 representative hotspot topologies from the two databases.

\subsection{Real Sample Topologies}

The topologies used in the first set of simulations are shown in Fig. 6 drawn from the real hotspot databases. Each edge in the figure represents the neighboring interference which means that an AP is in the carrier-sensing range of its connected APs. Therefore, when any neighbor APs of the AP are transmitting data, it senses the channel becoming busy. The first scenario in Fig. 6, for example, corresponds to the well-known FIM (Flow-In-the-Middle) topology. It was easy to find such topologies in real-world AP deployments. Every AP is configured to have 4 associated client nodes. Each client node performs an FTP download using TCP. Therefore, there is upstream and downstream traffic since TCP ACKs are transmitted from the clients to the AP. The simulation time is set to 60sec.

For each of these topologies, the throughput distribution of APs are plotted in Fig. 7. We compare the performance of our channel access control algorithm with 


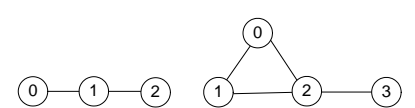

(a)

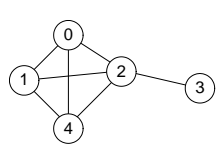

(d) (b)

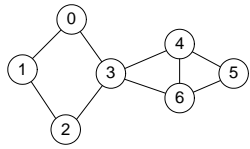

(e)

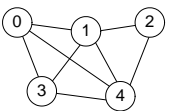

(c)

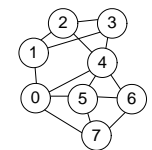

(f)

Figure 6: Simulated network topologies: 6 scenarios

those of the natural mode ('No control'). From the results of 'no control' mode in Fig. 7, we can observe severe throughput imbalance among APs in most scenarios. For example, $A P_{1}$ in scenario (a) and (c), $A P_{2}$ in scenario (b) and (d), and $A P_{5}$ in scenario (f) are shown to experience severe starvation. With the proposed scheme, the results indicate the improved throughput for the starving APs.

We study the effect of control level by using different $B I F S_{\max }$ for $B I F S_{\max }=$ 10 slots and $B I F S_{\max }=30$ slots, where a larger $B I F S_{\max }$ implies a higher possible restriction of the dominating AP's channel access thereby seeking for better fairness. To measure the improvement of fairness, we use Jain's fairness index, which is given by $\frac{\left(\sum_{i} x_{i}\right)^{2}}{n \cdot\left(\sum_{i} x_{i}^{2}\right)}$, where $x_{i}$ is the throughput of $B S S_{i}$ in each simulation. Note that Jain's fairness index lies between 0 and 1 and the closer to 1 , the fairer. From the results shown in Fig. 8, one can see that our scheme provides good fairness in most cases. A larger $B I F S_{\max }$ achieves a higher degree of fairness as shown in Fig. 7. However, we can see that there exists a tradeoff between fairness and throughput. In other words, a higher degree of fairness is achieved at the cost of throughput.

To understand the property of proposed scheme, we repeat the simulation for the scenario (c) in Fig. 6. In the simulation, the control module is turned on during $[25,40]$ seconds. Fig. 9 illustrates the effect of our channel access control algorithm. During this interval, we can see that the throughput of each AP is regulated by the cooperative channel management mechanism.

\subsection{Simulation in a Large-scale Network}

We evaluate the proposed scheme by applying it on a large-scale 802.11 network. A hexagonal topology consisting of BSSs is used for the simulation, where 


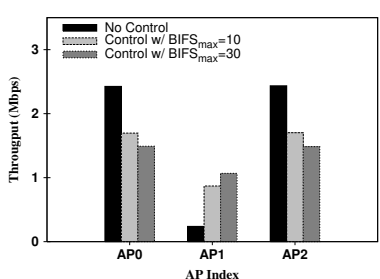

(a)

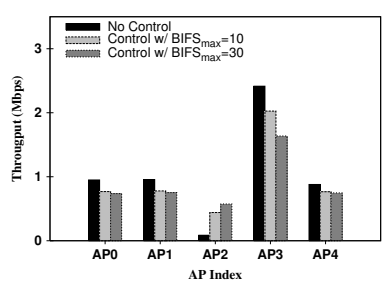

(d)

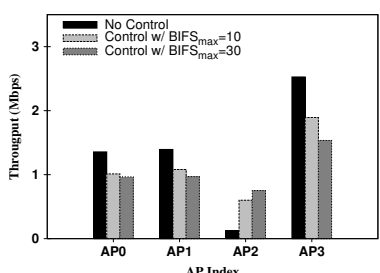

(b)

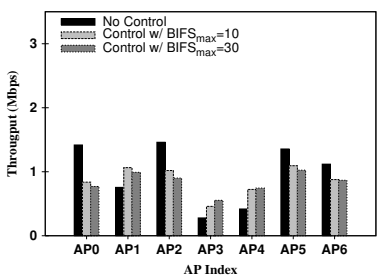

(e)

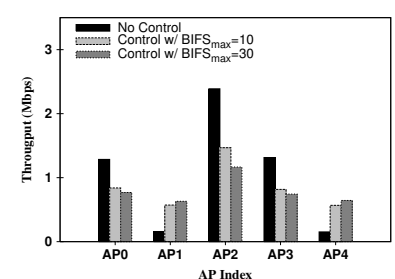

(c)

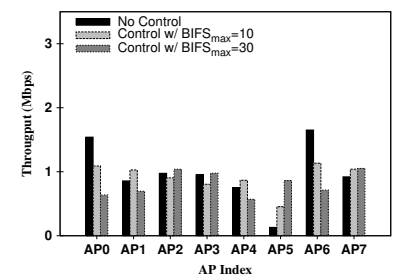

(f)

Figure 7: Throughput distribution of APs for 6 scenarios : Two control levels are considered $B I F S_{\max }=10$ and 30 slots

one AP is located in each BSS and comprises 10 clients. The distance between an AP and their clients is set to $25 \mathrm{~m}$. The traffic model is the same as the first scenario, i.e., each client node is performing an FTP download using TCP. The simulation results are plotted in Fig. 10. The throughput of each AP is represented as the size of circle. In Fig. 10(a), we can observe that the severe throughput imbalance among APs - almost a half of BSSs are shown to suffer from starvation. Fig. 10(b) shows the performance when the proposed scheme is adopted. From the result, we can see that our scheme eliminates the starving BSS that existed when the control is not employed.

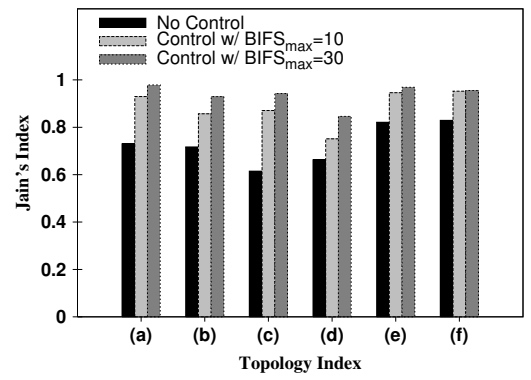

Figure 8: Fairness index for 6 scenarios 


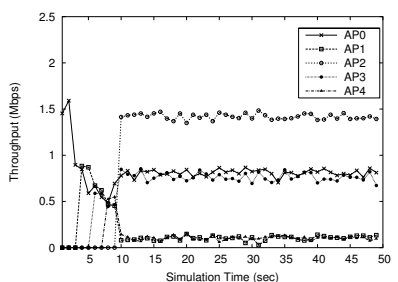

(a) No control

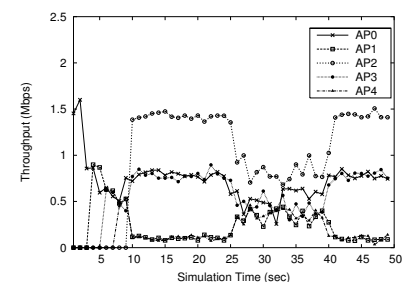

(b) With $\quad$ control
$\left(B I F S_{\max }=10\right.$ slots $)$

Figure 9: Throughput performance of each BSSs (control during [25,40]sec)

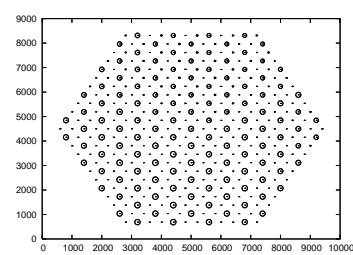

(a) Throughput distribution without control

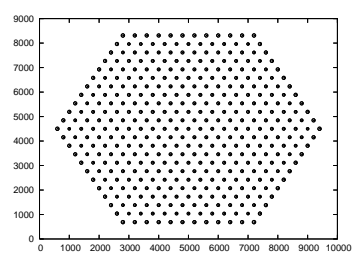

(b) Throughput distribution with control

Figure 10: Effect of dynamic rate control: (a) without control and (b) with control (40sec simulation)

\subsection{Voice-over-IP}

We evaluate the effectiveness of our approach for VoIP calls in dense WLANs. For a VoIP session, we use UDP packets of 160 bytes, where packets are continuously exchanged for the duration of the call. The traffic generating interval was set to $30 \mathrm{~ms}$ which is a typical voice frame packetization interval. We simulated a chain topology with 3 APs (the first scenario in Fig. 6-(a)). Two APs located at the edge have 4 stations where 2 stations use VoIP calls (bi-directional) and 2 stations have FTP flows. The AP in the middle has two clients using VoIP calls. In the simulation, the call deadline for VoIP was set to 250ms [29], i.e., if the VoIP traffic takes more than $250 \mathrm{~ms}$ to be deliverd at the destination, it is regarded as lost. The simulation results for QoS support through IEEE 802.11e are presented in Table 2. The loss rate of VoIP packets in two side APs were 0.036 and 0.029, respectively. On the other hand, the loss rate of VoIP packets through the middle AP were 1, i.e., all VoIP packets experienced extremely large latency and did not meet the deadline. With the control, as shown in Table 2, the loss rate of the starved BSS reduced to zero without much sacrifice of adjacent VoIP flows. 
Table 2: Loss rate through inter-AP control

\begin{tabular}{|c|c|c|c|}
\hline & $A P_{0}$ & $A P_{1}$ & $A P_{2}$ \\
\hline Loss Rate w/o control & 0.036 & 1.000 & 0.029 \\
Loss Rate w/ control & 0.046 & 0.000 & 0.031 \\
\hline
\end{tabular}

\section{Conclusion}

In this paper, we proposed a new way of enhancing the IEEE 802.11e to provide QoS support in large-scale WLANs with high inter-AP channel contention. We also presented a management mechanism that can resolve the throughput imbalance among BSSs. Our approach can be viewed as an extension of QoS provisioning from a single-WLAN domain to a multi-WLAN domain, i.e., a complementary approach to enhancing the 802.11e standard. Our performance evaluation has shown that the proposed inter-AP differentiation-based QoS management overcomes the limitation of legacy 802.11e in densely-deployed multi-AP WLANs.

\section{Acknowledgment}

This work was supported by the National Research Foundation of Korea Grant funded by the Korean Government(Ministry of Education, Science and Technology) [KRF-2008-357-D00219].

\section{References}

[1] IEEE, Part 11: Wireless LAN Medium Access Control (MAC) and Physical Layer (PHY) Specifications. IEEE Std 802.11-1999, 1999.

[2] IEEE, Draft Supplement to Part 11: Wireleess Medium Access control (MAC) and physical (PHY) specifications: Medium Access Control (MAC) Enhancements for Quality of Service(QoS), IEEE Std 802.11e/D4, 2003.

[3] The network simulator - ns2. http://www.isi.edu/nsnam/ns/, online link.

[4] http://www.wigle.net/, online link.

[5] S. Agarwal, J. Padhye, V. N. Padmanabhan, L. Qiu, A. Rao, and B. Zill, “ Estimation of link interference in static multi-hop wireless networks," in Proc. Internet Measurement Conference (IMC), Oct. 2005. 
[6] N. Ahmed, U. Ismail, S. Keshav, and K. Papagiannaki, "Online Estimation of RF Interference,' In Proc. ACM CoNext'08, 2008.

[7] N. Ahmed and S. Keshav, "SMARTA: a self-managing architecture for thin access points," in Proc. CoNext, Dec. 2006.

[8] A. Akella, G. Judd, S. Seshan, and P. Steenkiste, "Self-Management in Chaotic Wireess Deployments," Wireless Networks Journal(WINET), Special Issue on Selected Papers from MobiCom 2005, vol. 13, no. 6, pp. 737-755, Dec. 2007.

[9] A. Balachandran, G. Voelker, and P. Bahl, "Wireless hotspots: Current challenges and future directions," Mobile Networks and Applications, vol. 10, no. 3, pp. 265 - 274, June, 2005.

[10] Y. Bejerano, S. Han, and L. Li, "Fairness and load balancing in wireless lans using association control," in Proc ACM Mobicom'04, Sep., 2004.

[11] G. Bianchi, I. Tinnirello, and L. Scalia, "Understanding 802.11e ContentionBased Prioritization Mechanisms and Therir Coexistence with Legacy 802.11 Stations," IEEE Network, 2005.

[12] F. Cali, M. Conti and E. Gregori, "Dynamic Tuning of the IEEE 802.11 Protocol to Achieve a Theoretical Throughput Limit," IEEE/ACM Trans. on Networking (TON), vol. 8, pp.785-799, 2000.

[13] C. Chaudet, I. G. Lassous, and B. Gaujal. "Study of the Impact of Asymmetry and Carrier Sense Mechanism in IEEE 802.11 Multi-hops Networks through a Basic Case," in Proc. PE-WASUN'04, Venice, Italy, Oct., 2004.

[14] D. Chiu and R. Jain, "Analysis of the Increase and Decrease Algorithms for Congestion Avoidance in Computer Networks," Journal of Computer Networks and ISDN,vol. 17, no. 1, pp. 1-14, 1989.

[15] S. Floyd, “Congestion control principles,” RFC 2914, Sep. 2000.

[16] M. Garetto, T. Salonidis, and E. W. Knightly, "Modeling Per-flow Throughput And Capturing Starvation In CSMA Multi-hop Wireless Networks," in Proc. IEEE INFOCOM'06, Mar., 2006.

[17] J. Geier, “Assigning 802.11b Access Point Channels,” In WiFi planet, 2002. 
[18] M. Heusse, F. Rousseu, R. Guilier, and A. Duda, "Idle Sense: An Optimal access Method for high throughput and Fairness in Rate diverse wireless LANs," in Proc. ACM SIGCOMM'05, pp. 121-132, 2005.

[19] A. Hills, "Large-scale wireless LAN design," IEEE Communications Magazine, 39(11):98-107, 2001.

[20] C. Hua and R. Zheng, "Starvation Modeling and Identification in Dense 802.11 Wireless Community Networks," in Proc. IEEE INFOCOM'08, Apr., 2008.

[21] C. Hun, and J. C. Hou, "A Novel Approach to Contention Control in IEEE 802.11e-Operated WLANs," in Proc. IEEE INFOCOM'07, May, 2007.

[22] B. Kauffmann, F. Baccelli, A. Chainteau, V. Mhatre, K. Papagiannaki, and C. Diot. "Measurement-Based Self Organization of Interfering 802.11 Wireless Access Networks,", in Proc. IEEE INFOCOM'07, May, 2007.

[23] Y. Jian, and S. Chen, "Can CSMA/CA networks be made fair?," in Proc. ACM Mobicom'08, Sep., 2008.

[24] D. Kotz. CRAWDAD data set Dartmouth college. http://crawdad.cs.dartmouth.edu/.

[25] J. Lee, W. Kim, S. Lee, D. Jo, J. Ryu, T. Kwon, and Y. Choi, "An Experimental Study on the Capture Effect in 802.11a Networks," in Proc. ACM WINTECH'07, Sep., 2007.

[26] V. Mhatre, K. Papagiannaki, and F. Baccelli, "Interference mitigation through power control in high density 802.11 WLANs," in Proc IEEE INFOCOM'07, May, 2007.

[27] A. Mishra, S. Banerjee, and W. Arbaugh, "Weighted coloring based channel assignment for wlans," Mobile Computer Communications Review (MC2R), vol. 9, no. 3, pp. 19-31, July, 2005.

[28] A. Mishra, V. Shrivastava, D. Agarwal, and S. Banerjee, "Distributed Channel Management in Uncoordinated Wireless Environments," in Proc. ACM MOBICOM'06, 2006.

[29] V. Namboodiri, and L. Gao, "Towards Energy Efficient VoIP over Wireless LANs," in Proc. ACM Mobihoc'08, Sep., 2008. 
[30] I. Papanikos and M. Logothetis, "A study on dynamic load balance for IEEE 802.11b wireless LAN," in Proc. COMCON'01, June, 2001.

[31] D. Qiao and K. Shin, "Achieving efficient channel utilization and weighted fairness for data communications in IEEE 802.11 WLAN under the DCF," in Proc. of International Workshop on Quality of Service, May 2002.

[32] L. Qiu, Y. Zhang, F. Wang, M. K. Han, and R. Mahajan, "A general model of wireless interference," in Proc. ACM MOBICOM'07, Sept. 2007.

[33] C. Reis, R. Mahajan, M. Rodrig, D. Wetherall, and J. Zahorjan, "Measurement-based models of delivery and interference," in Proc. ACM SIGCOMM'06, Sept. 2006.

[34] E. Rozner, Y. Mehta, A. Akella and L. Qiu, "Traffc-Aware Channel Assignment in enterprise Wireless LANs," in Proc. IEEE ICNP'07, Oct., 2007.

[35] V. Shrivastava, N. Ahmedand S. Rayanchu, S. Banerjee, S. Keshav, K. Papagiannaki, and A. Mishra, "CENTAUR: realizing the full potential of centralized wlans through a hybrid data path,", in Proc ACM MobiCom '09, Sept., 2009.

[36] A. Vasan, R. Ramjee, and T. Woo, "ECHOS: Enhanced capacity 802.11 hotspots," in Proc. IEEE INFOCOM'05, Mar., 2005.

[37] X. Wang and K. Kar. "Throughput Modelling and Fairness Issues in CSMA/CA Based Ad-Hoc Networks," in Proc. IEEE INFOCOM'05, Mar., 2005.

[38] Y. Yang and R. Kravets, "Thoughput guarantees for multi-priority traffic in ad hoc networks," in Proc. of IEEE MASS, 2005. 